\title{
Nanocellulosic fillers for waterborne wood coatings: reinforcement effect on free-standing coating films
}

\author{
Marcel Kluge $^{1,2} \cdot$ Stefan Veigel $^{2} \cdot$ Stefan Pinkl $^{3} \cdot$ \\ Ute Henniges $^{4}$ - Cordt Zollfrank $^{5}$ - Albert Rössler ${ }^{6}$. \\ Wolfgang Gindl-Altmutter ${ }^{2}$ (D)
}

Received: 15 June 2016/Published online: 10 February 2017

(C) The Author(s) 2017. This article is published with open access at Springerlink.com

\begin{abstract}
Coatings fulfill an important function in providing functionality and service life to wood surfaces. In the present study, the potential of nanocellulosic fillers toward improving waterborne wood coating mechanics is evaluated using free-standing coating films. At $2 \%$ filler content, significant improvements in static and dynamic mechanical properties were observed. The extent of these improvements was different depending on whether high-aspect-ratio cellulose nanofibrils of short cellulose nanocrystals were used. Chemical surface modification of cellulose nanofibrils did not provide further improvement. The water-vapor sorption properties of the coating films, which were also evaluated, did not show significant effects due to addition of nanocellulose, while optical transparency slightly decreased.
\end{abstract}

Wolfgang Gindl-Altmutter

wolfgang.gindl@boku.ac.at

1 Fraunhofer Institute for Wood Research - Wilhelm-Klauditz-Institut WKI, Braunschweig, Germany

2 Department of Material Sciences and Process Engineering, BOKU - University of Natural Resources and Life Sciences Vienna, Tulln, Austria

3 Kompetenzzentrum Holz GmbH, Linz, Austria

4 Department of Chemistry, BOKU - University of Natural Resources and Life Sciences Vienna, Tulln, Austria

5 Chair of Biogenic Polymers, Straubing Center of Science, Technical University of Munich, Munich, Germany

6 ADLER-Werk Lackfabrik Johann Berghofer GmbH \& Co, Schwaz, Austria 


\section{Introduction}

The exposure of wood surfaces to external effects such as moisture, heat, ultraviolet radiation or mechanical stress can result in a deterioration of its properties. This can have an effect on the appearance, but may also lead to a degradation of the material and consequently a loss of function. Therefore, coatings are applied to enhance the durability and thus extend the service life of surfaces. Depending on the intended use and possible contact to stress factors, a coating material has to satisfy various requirements. In addition to good resistance to heat and chemicals, coatings typically must be highly resistant to scratches, abrasion and impact, for example falling objects. The mechanical performance of a coating is determined by the chemistry of the used binder.

One approach to enhance the functionality and thus the end user value of coatings is the addition of nanoparticles (Hincapie et al. 2015; Hischier et al. 2015; Nikolic et al. 2015; Pilotek and Tabellion 2005; Weiss 1997). Due to their morphology, they have a very large surface-to-volume ratio, which allows them to interact intensively with their surroundings and therefore enhance the mechanical properties of a polymer matrix. Additionally, their nanosize ensures that transparency is largely preserved. As a result of more severe environmental restrictions, solvent-based coating materials are being progressively replaced by alternatives that contain low amounts of volatile compounds. In the woodworking sector, the application of waterborne coating materials is a widely used practice to meet these requirements (Philipp 2010). Nevertheless, solvent-based coating systems are still extensively used, for example, in the furniture industry, since the performance of their waterborne counterparts shows potential for further improvements (Challener 2015; Philipp 2010).

One unique distinguishing feature of the woodworking industry in the context of other industries is the bio-based and renewable character of its lead resource wood. Therefore, bio-based solutions for property improvements are particularly welcome in this sector. While inorganic nanoparticles have already been implemented in various coating solutions, the addition of bio-based nanofillers is still at an early stage, being studied mostly at the laboratory level.

Cellulosic nanofillers (nanocellulose) are being intensely discussed in the literature due to their excellent reinforcement potential in polymers (Eichhorn et al. 2010; Klemm et al. 2011; Lee et al. 2014; Moon et al. 2011). Nanocellulose may either be used in the form of long fibrils termed cellulose nanofibrils (CNF) produced by mechanical fibrillation of wood pulp, or in the shape of cellulose nanocrystals (CNC), which are produced by an additional acid hydrolysis step, resulting in higher crystallinity and lower aspect ratio compared to CNF. Since cellulose is essentially hydrophilic in terms of surface chemistry, it disperses well in aqueous media, whereas hydrophobization by means of chemical surface modification may be required to achieve homogeneous dispersion in nonpolar organic solvents or polymers (Habibi 2014). With regard to wood coatings, a number of studies have been published. CNC were reported to improve resistance against scratching and abrasion of acrylic latex, UV-curing waterborne varnish and high- 
solids coating (Kaboorani et al. 2016; Landry and Blanchet 2011; Poaty et al. 2014; Vardanyan et al. 2014, 2015; Veigel et al. 2014). In addition to improvements in coating mechanics, also optical effects may be imparted by $\mathrm{CNC}$ addition to coatings (Vlad-Cristea et al. 2013). Generally, straightforward dispersion of CNC in waterborne formulations is reported, whereas chemical hydrophobization, for example by means of an organofunctional silane (Landry and Blanchet 2011) or acryloyl chloride or alkyl quaternary ammonium bromides (Poaty et al. 2014), was required for nonpolar systems. Addition of CNF to coatings also provided improvements in mechanics (Grüneberger et al. 2014a; Veigel et al. 2014) and exhibited beneficial effects on the dispersion of inorganic additives (Grüneberger et al. 2015; Jämsä et al. 2011).

Besides effects on coating mechanics and dispersion of non-cellulosic additives, significant effects of nanocellulosic filler on the rheology of coatings were reported (Grüneberger et al. 2014b), which may lead to a replacement of current rheology modifiers by nanocellulose (Phipps et al. 2013).

In the present study, results on the static and dynamic mechanical behavior as well as the water sorption properties and optical transparency of free-standing films with different nanocellulosic fillers are shown. Besides the choice of nanocellulosic filler, also its surface chemistry is varied by means of acetylation. Here, the hypothesis that even though nanocellulose disperses well in waterborne coatings, its adhesion to the polymer component of the coating, which is nonpolar, may benefit from reduced polarity due to acetylation.

\section{Materials and methods}

The waterborne one-component varnish Aqua-Resist G50 (ADLER-Werk Lackfabrik Johann Berghofer GmbH \& Co KG, Austria) was used as coating material. This coating is suitable for indoor use, in particular for the surface finishing of wooden furniture and interior fittings. It is based on an acrylic binder and delivered with a solid content of approximately 34.5\%. Cellulose nanofibrils and nanocrystals (CNF and CNC, Fig. 1) were purchased from the University of Maine Process Development Center (http://umaine.edu/pdc/facilities-available-for-use/nanocellulosefacility/). The material is produced by mechanical disintegration of bleached softwood Kraft pulp without any additional pre-treatment. Both were obtained in the form of an aqueous slurry with a solid content of approximately 3 and 7\%, respectively. While the size distribution of $\mathrm{CNC}$ is fairly homogeneous, also larger micronsized aggregates are occasionally found in CNF (Fig. 1c). Acetic acid (100\%), acetic acid anhydride $(\geq 99 \%)$, acetone $(\geq 99.5 \%)$, ethanol $(\geq 99.8 \%)$, hydrochloric acid (0.5 N volumetric standard solution) and pyridine ( $\geq 99 \%)$ were obtained from Carl Roth $\mathrm{GmbH}+\mathrm{Co}$. KG, Germany. Sodium hydroxide $(0.1 \mathrm{~N}$ concentrate for standard solution) was purchased from Sigma-Aldrich Handels GmbH, Austria. All chemicals were used as received without further purification. High-purity water was used throughout. 

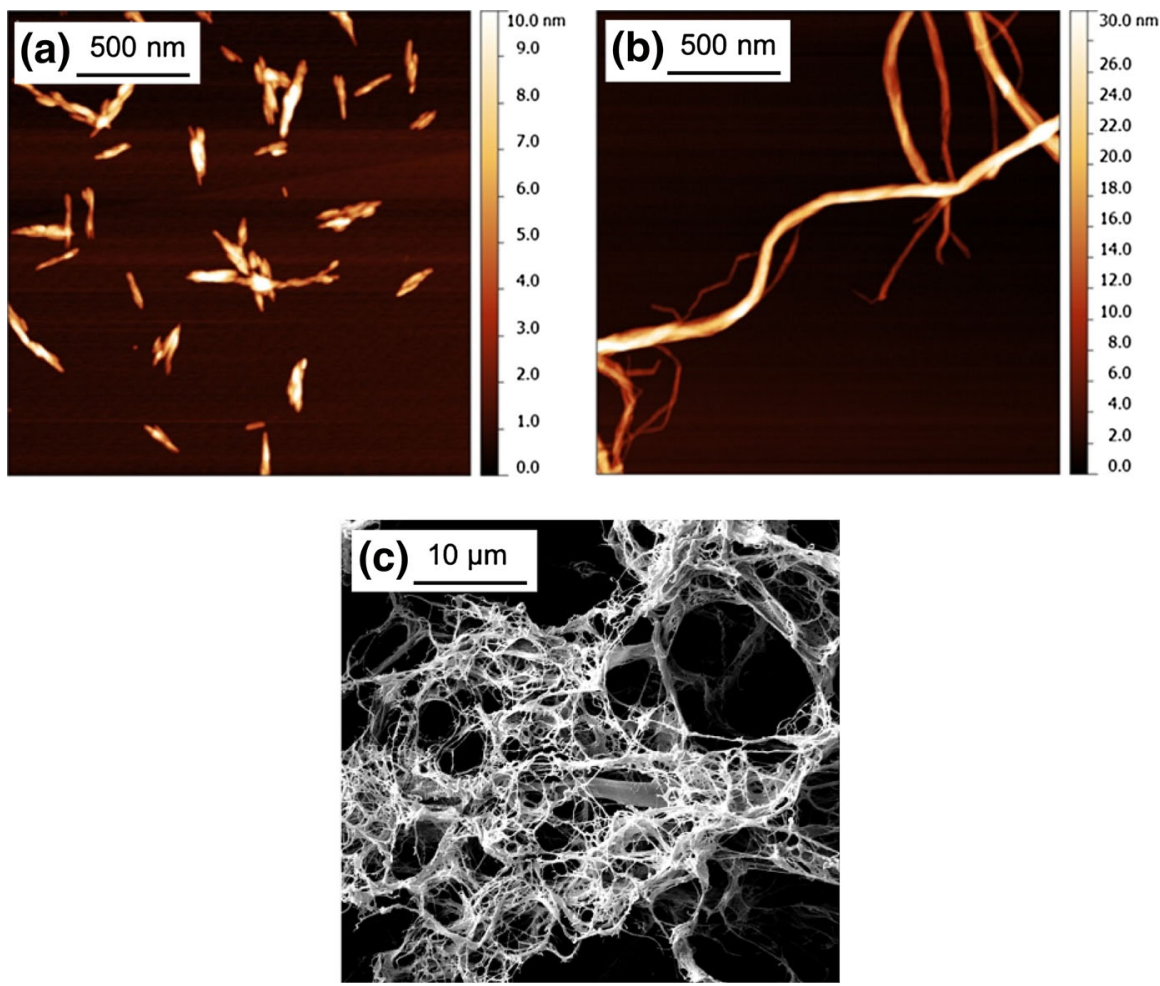

Fig. 1 Representative atomic force microscope (AFM) topography images of a cellulose nanocrystals, b nanofibrils and c SEM image of nanofibrils showing larger aggregates

\section{Acetylation of cellulose nanofibrils}

The acetylation of CNF was done according to protocols published in the literature (Ashori et al. 2014; Ernest-Saunders et al. 2014; Jonoobi et al. 2010). As a first step, a solvent exchange from water to a mixture of acetone and methanol (2:1) was carried out. Subsequently, CNF were Soxhlet extracted for $3 \mathrm{~h}$ at approximately $57.5^{\circ} \mathrm{C}$, using the same mixture of solvents to remove impurities and to increase the swelling of the fibers. Thereafter, CNF were solvent-exchanged to glacial acetic acid and refrigerated overnight. An aliquot containing approximately $5 \mathrm{~g}$ of cellulose nanofibrils was transferred into a two-neck round-bottom flask, subsequently adding acetic acid anhydride and acetic acid (in order to adjust viscosity) and pyridine. A typical batch contained $1 \%$ MFC, $31 \%$ acetic acid, $65 \%$ acetic anhydride and 3\% pyridine on a mass basis. The mixture was then heated to the reaction temperature of $100{ }^{\circ} \mathrm{C}$ where it was kept for $8.5 \mathrm{~h}$ under reflux. After cooling, the treated CNF were washed with purified water until a neutral $\mathrm{pH}$ was obtained, followed by Soxhlet extraction with water. Finally, potential aggregates were disintegrated with an ultrasonic bath for $30 \mathrm{~min}$ and the product was refrigerated until further usage. Acetylated CNF is termed CNFac in the following text. 


\section{Determination of degree of substitution}

A heterogeneous saponification method was applied to determine the acetyl content. Briefly, the material was de-watered, freeze-dried and milled with a swing mill that was cooled by liquid nitrogen (CryoMill, Retsch $\mathrm{GmbH}$, Germany). The resulting powder was dried in a drying cabinet at $105^{\circ} \mathrm{C}$ for $2 \mathrm{~h}$ to remove residual water. Three hundred and seventy-five milligrams of cellulose powder was transferred into a 100 -mL Erlenmeyer flask, $15 \mathrm{~mL}$ of ethanol (75\%) was added, and the mixture was sonicated for $30 \mathrm{~min}$ at $60{ }^{\circ} \mathrm{C}$. Sonication was repeated for another $15 \mathrm{~min}$ after adding $15 \mathrm{~mL}$ of $0.5 \mathrm{~N} \mathrm{NaOH}$ solution. Residual $\mathrm{NaOH}$ was back-titrated with $0.5 \mathrm{~N} \mathrm{HCl}$ after $48 \mathrm{~h}$, using phenolphthalein as indicator. An additional milliliter of $\mathrm{HCl}$ was added, and the mixture was left to stand overnight that remaining $\mathrm{NaOH}$ could diffuse from the cellulose. $0.5 \mathrm{~N} \mathrm{NaOH}$ solution was then used to titrate the excess $\mathrm{HCl}$. Titration of the sample and a blank was done in triplicate. The percentage of acetyl groups per anhydroglucose unit and the corresponding DS were calculated according to Samios et al. (1997).

\section{Fourier transform infrared spectroscopy (FTIR)}

Powder of acetylated and untreated CNF was scanned in absorbance mode with a mid-infrared spectrometer using an ATR-unit (PerkinElmer Inc., USA). Fifty scans were recorded at a resolution of $4 \mathrm{~cm}^{-1}$ within the range of $4000-650 \mathrm{~cm}^{-1}$. The contact surface had a dimension of $2 \mathrm{~mm}^{2}$, and a pressure of $0.1 \mathrm{Nm}$ was applied. The spectra were normalized to the intensity of the band at $2890 \mathrm{~cm}^{-1}$ corresponding to $\mathrm{C}-\mathrm{H}$ stretching, where no shift is expected due to acetylation (Ernest-Saunders et al. 2014).

\section{Preparation of free-standing nanocomposite coating films}

Mixtures of coating material and nanoparticles in water were prepared by mixing nanocellulose dispersion with waterborne coating at high shear rates (Ultra-Turrax T8 with dispersing element $\mathrm{S} 8 \mathrm{~N}-5 \mathrm{~N}$, IKA-Werke, Germany). The target nanocellulose content was set to 0.5 and $2 \%$ mass, respectively, based on the coating materials solid content. The mixtures were degassed using a rotary evaporator. A rotational rheometer (CVO 50, Bohlin Instruments Ltd, United Kingdom) with a cone-plate measuring system $(4 \% 40 \mathrm{~mm})$ was used for viscosity measurements. All tests were done at a temperature of $20^{\circ} \mathrm{C}$, a constant shear rate of $5 \mathrm{~s}^{-1}$ and a gap of $150 \mu \mathrm{m}$. The sample size was $1.3 \mathrm{~mL}$, and at least three specimens were tested for each type of coating material. Five values were obtained for each measurement. Free-standing coating films were produced by casting the mixtures on a Teflon mold which had a recess with a height of $400 \mu \mathrm{m}$. A glass specimen holder was used to remove excess material and to smoothen the surface. Water was evaporated at room temperature, and subsequently, the films were carefully peeled off. For further drying, the films were placed between sheets of paper to prevent contamination and warping. Prior to testing, they were stored at standard climate $\left(20{ }^{\circ} \mathrm{C}\right.$, relative humidity of $\left.65 \%\right)$ for 7 days. Coating films were 
cut to size with a razor blade, and their thickness was measured with a digital dial indicator.

\section{Characterization of free-standing nanocomposite coating films}

Tensile tests were done with a universal testing machine (Z020, Zwick Roell AG, Germany) at a speed of $10 \mathrm{~mm} \mathrm{~min}^{-1}$ using a $500 \mathrm{~N}$ load cell. Rectangular specimens with dimensions of $8 \times 70 \mathrm{~mm}^{2}$ and thickness between 78 and $112 \mu \mathrm{m}$ were tested. The clamping length was $40 \mathrm{~mm}$. At least twenty specimens were tested per type until fracture.

The viscoelastic properties of coating films were determined by dynamic mechanical analysis (DMA 242 C, Netzsch-Gerätebau GmbH, Germany). Experiments were done in tension mode with maximum amplitude of $30 \mu \mathrm{m}$ $(0.3 \%$ strain of free length) and a frequency of $1 \mathrm{~Hz}$. A static force of $0.2 \mathrm{~N}$ was maintained, while the dynamic force was set to a maximum value of $0.5 \mathrm{~N}$. Tests were done in a nitrogen atmosphere in a dynamic temperature range of -50 to $150{ }^{\circ} \mathrm{C}$ with a heating rate of $2 \mathrm{~K} \mathrm{~min}^{-1}$. A minimum of four replicates with a rectangular geometry of $5 \times 25 \mathrm{~mm}^{2}$ and a clamping length of $10 \mathrm{~mm}$ was tested.

To assess the potential influence of nanocellulose on the water uptake of coating films, sorption isotherms were recorded by means of dynamic vapor sorption (DVS Advantage 1, Surface Measurement Systems Ltd., UK). Small pieces of films with equivalent mass between 9.88 and $12.67 \mathrm{mg}$ were used. A constant temperature of $25{ }^{\circ} \mathrm{C}$ was maintained.

Transmission spectra of selected films were characterized by means of a PerkinElmer Lambda $35 \mathrm{UV} /$ Vis spectrometer equipped with a solid sample holder, using a scan rate of $480 \mathrm{~nm} \mathrm{~min}{ }^{-1}$. Transparency of the films was determined at a wavelength of $600 \mathrm{~nm}$.

\section{Results and discussion}

\section{Chemical modification}

FTIR spectra shown in Fig. 2 indicate significant changes in the chemistry of CNF after acetylation. Three characteristic bands are related to acetyl groups and therefore acetylated cellulose: the $\mathrm{C}=\mathrm{O}$ stretching of carbonyl groups in the ester bonds around $1745-1740 \mathrm{~cm}^{-1}$, the vibration peaks at $1240-1235 \mathrm{~cm}^{-1}$ due to stretching of $\mathrm{C}-\mathrm{O}$ bonds and the methyl in-plane bending of $\mathrm{C}-\mathrm{CH}_{3}$ around $1375 \mathrm{~cm}^{-1}$ (Ashori et al. 2014; Cetin et al. 2009; Higgins et al. 1961; Jonoobi et al. 2010). All of them could be easily identified in the spectrum obtained for the modified CNF. On the other hand, no distinct peak typical for carboxylic groups was found around $1700 \mathrm{~cm}^{-1}$, which shows that unreacted free acetic acid was efficiently removed from the modified material during washing (Jonoobi et al. 


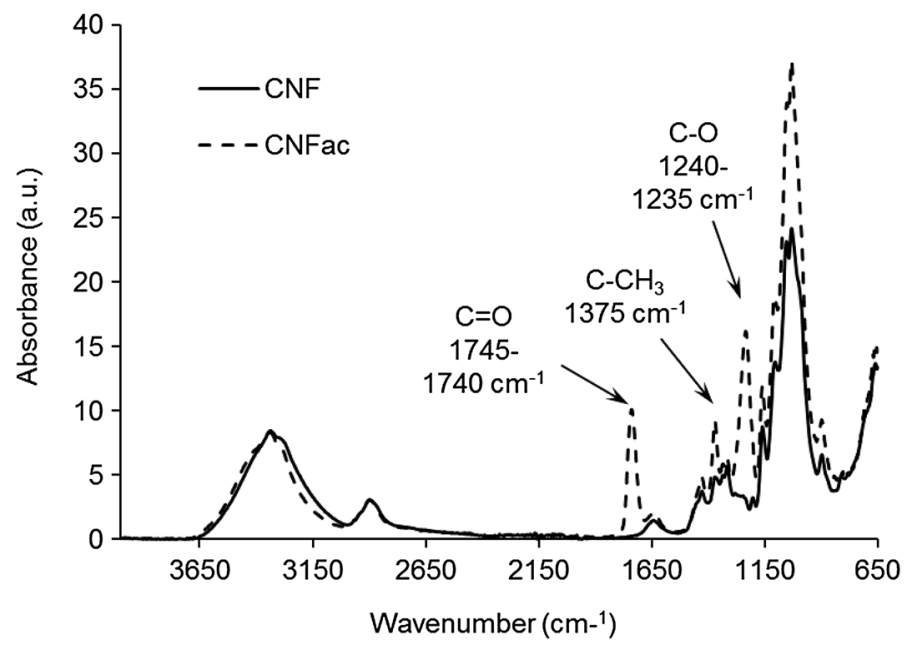

Fig. 2 FTIR spectra of unmodified and acetylated CNF

2010). A moderate but significant average degree of substitution of 0.51 was obtained from duplicate determinations.

\section{Mechanics of free-standing coating films}

An increase in the coating material's viscosity was observed at the addition of cellulosic nanoparticle in good agreement with the literature (Grüneberger et al. 2014b; Xu et al. 2013). This was true in particular at a content of 2\% (Table 1). Viscosity is an important processing parameter as it may limit the sprayability of a coating formulation and may also affect the spreading of a coating across a surface. In the context of the present study, viscosity was not a limiting factor. Furthermore, it is known that nanocellulose suspensions show shear thinning (Grüneberger et al. 2014b; Iotti et al. 2011; Li et al. 2015), which may facilitate processing even at higher initial viscosity.

The results of tensile tests are shown in Fig. 3 and Table 2. Overall, the coating films showed moderate strength and stiffness at high extensibility $>100 \%$. These characteristics were not significantly affected by addition of $0.5 \%$ nanocellulose,

Table 1 Dynamic viscosity and solid content (SC) of coating material variants (standard deviation in parentheses, four repetitions each)

\begin{tabular}{lll}
\hline Formulation & Viscosity (Pa s) & Solid content $(\%)$ \\
\hline Reference & $1.33( \pm 0.009)$ & 34.39 \\
$0.5 \% \mathrm{CNC}$ & $1.65( \pm 0.011)$ & 33.75 \\
$0.5 \% \mathrm{CNF}$ & $2.02( \pm 0.051)$ & 32.50 \\
$0.5 \% \mathrm{CNFac}$ & $1.81( \pm 0.017)$ & 32.50 \\
$2 \% \mathrm{CNC}$ & $2.63( \pm 0.021)$ & 26.84 \\
$2 \% \mathrm{CNF}$ & $2.20( \pm 0.064)$ & 23.28 \\
$2 \% \mathrm{CNFac}$ & $2.12( \pm 0.064)$ & 23.28 \\
\hline
\end{tabular}


(a)

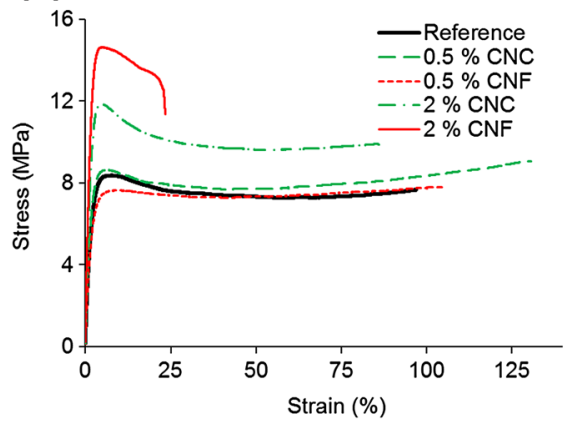

(b)

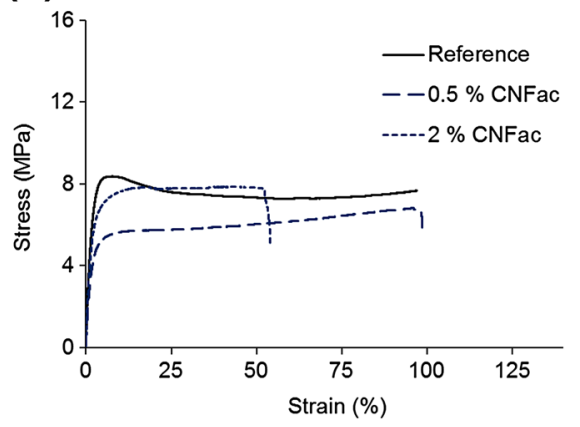

Fig. 3 Representative stress-strain curves of tensile tests with free-standing coating films comparing $\mathbf{a}$ unmodified and $\mathbf{b}$ acetylated filler

Table 2 Results of tensile tests with free-standing coating films (standard deviation in parentheses, 20 repetitions each)

\begin{tabular}{lclc}
\hline Type of sample & Tensile strength $(\mathrm{MPa})$ & Elastic modulus $(\mathrm{MPa})$ & Failure strain $(\%)$ \\
\hline Reference & $8.57( \pm 1.51)$ & $389( \pm 128)$ & $103( \pm 45)$ \\
$0.5 \% \mathrm{CNC}$ & $8.96( \pm 1.52)$ & $410( \pm 123)$ & $126( \pm 33)$ \\
$0.5 \% \mathrm{CNF}$ & $7.95( \pm 0.85)$ & $371( \pm 74)$ & $108( \pm 19)$ \\
$0.5 \% \mathrm{CNFac}$ & $7.04( \pm 0.73)$ & $267( \pm 84)$ & $100( \pm 15)$ \\
$2 \% \mathrm{CNC}$ & $11.76( \pm 1.3)$ & $635( \pm 89)$ & $86( \pm 26)$ \\
$2 \% \mathrm{CNF}$ & $14.91( \pm 1.53)$ & $801( \pm 99)$ & $23( \pm 9)$ \\
$2 \% \mathrm{CNFac}$ & $7.84( \pm 1.02)$ & $376( \pm 87)$ & $50( \pm 11)$ \\
\hline
\end{tabular}

whereas addition of $2 \%$ nanoparticles resulted in clear changes. A strong increase in strength and stiffness was observed with $2 \% \mathrm{CNF}$, while at the same time extensibility was drastically reduced. Similarly, but to a lesser extent, strength and stiffness also increased with $2 \% \mathrm{CNC}$, while extensibility of these films was largely preserved. This overall trend, i.e., increased strength and stiffness with simultaneously reduced extensibility, agrees well with reports in the literature on CNFreinforced coatings (Grüneberger et al. 2014a) and is also valid for inorganic platelet-reinforced coatings (Nikolic et al. 2015). For CNF, such an effect is proposed to be due to well-known percolation phenomena, which are correlated with the length and content of nanocellulosic filler in polymers (Azizi Samir et al. 2005; Bulota et al. 2012; Littunen et al. 2013; Xu et al. 2013). At constant filler content, short $\mathrm{CNC}$ are less prone to network formation than high-aspect-ratio CNF. Network formation benefits the strength and stiffness of nanocellulose polymers, but severely limits their extensibility. As for acetylated variants, no improvement in film properties was observed. By contrast, film performance degraded with addition of CNFac. Presumably, hydrophobization of this material led to inhomogeneous dispersion in the polymer matrix entailing reduced mechanical performance. Extensibility is crucial in wood coatings, which are required to follow movement of 
(a)

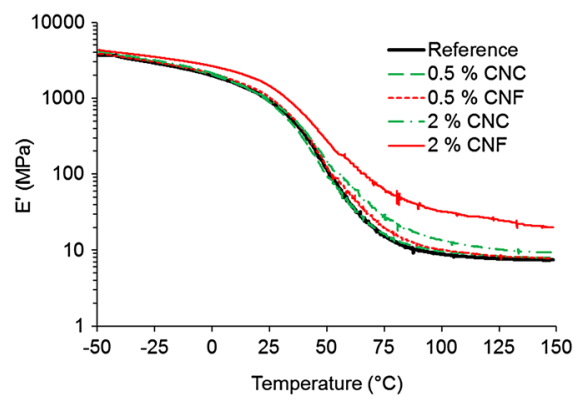

(b)

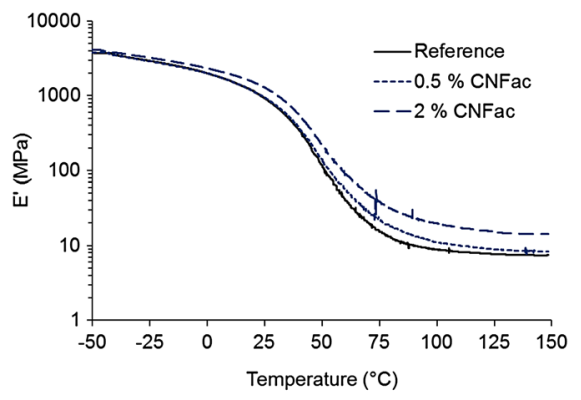

Fig. 4 Representative results for the storage modulus of free-standing coating films obtained by DMA comparing a unmodified and $\mathbf{b}$ acetylated filler

(a)

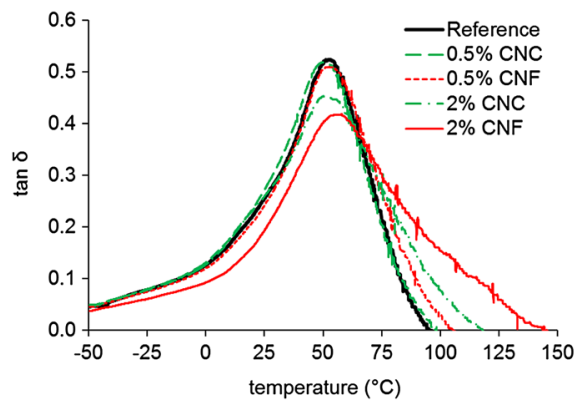

(b)

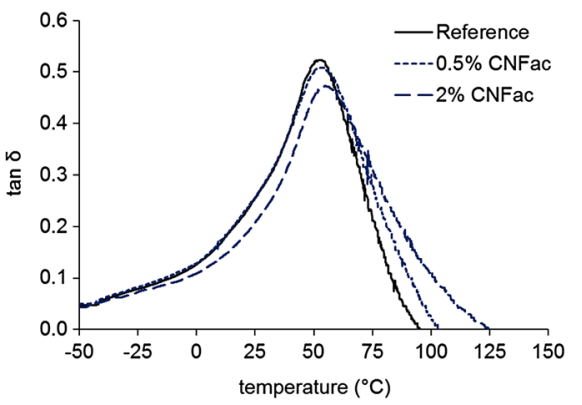

Fig. 5 Representative results for the loss tangent of free-standing coating films obtained by DMA comparing a unmodified and $\mathbf{b}$ acetylated filler

the wood substrate due to hygroexpansion. Therefore, small aspect ratio is probably advantageous with regard to an optimal balance between reinforcement efficiency and preservation of extensibility.

The general trends observed in static tensile tests (Fig. 3; Table 2) were confirmed by the results of DMA (Figs. 4, 5). While the ratio between the storage moduli in the glassy and rubbery states is hardly affected at $0.5 \%$ nanocellulose content, clear changes toward higher stiffness in the rubbery region are seen at $2 \%$ filler content for both CNF and CNFac (Fig. 4). Additionally, also the loss tangent of these variants significantly differs, with $T_{\mathrm{g}}$ shifting toward higher temperatures. For the variant with $2 \% \mathrm{CNF}, T_{\mathrm{g}}$ shifted from $52{ }^{\circ} \mathrm{C}$ (Reference) to $56{ }^{\circ} \mathrm{C}$, and for the variant with $2 \%$ acetylated $\mathrm{CNF}$, a shift to $54{ }^{\circ} \mathrm{C}$ was determined. Contrarily, no statistically significant changes in $T_{\mathrm{g}}$ were observed for lower CNF content nor any of the CNC variants. Again these observations may be explained by network formation of CNF as elucidated comprehensively in the literature (Azizi Samir et al. 2005; $\mathrm{Xu}$ et al. 2013). Similar trends are frequently reported for the dynamic mechanical analysis of cellulose nanocomposites. While a decrease in the peak height is generally observed with increasing filler content, a broadening and shift of 
(a)

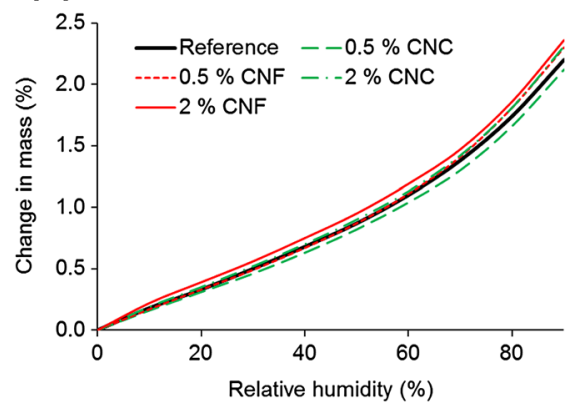

(b)

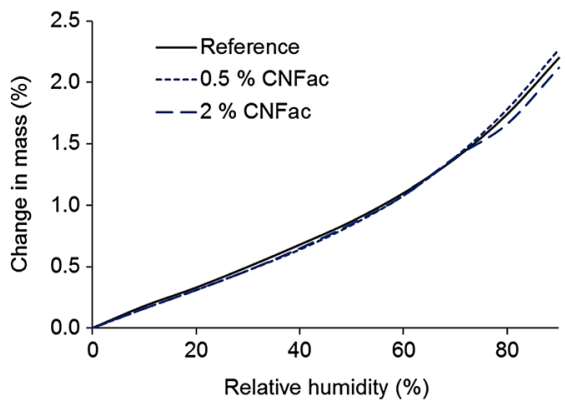

Fig. 6 Representative sorption isotherms of free-standing coating films obtained by DVS comparing a unmodified and $\mathbf{b}$ acetylated filler

$\tan \delta$ is only partially found (Ben Elmabrouk et al. 2009; Besbes et al. 2011; Favier et al. 1995).

\section{Water vapor sorption and optical transparency}

Cellulose is a hydrophilic polymer and cellulosic materials adsorb water vapor up to $30 \%$ of their dry mass (Belbekhouche et al. 2011; Hill et al. 2009; Xie et al. 2011), whereas water sorption of acrylic coating systems is much less pronounced (Liu et al. 2015). This aspect is often raised in discussions with producers of coating formulations, who in view of the hydrophilicity of nanocellulose fear a reduction in water repellency or stability against liquid water of coating formulations due to addition of nanocellulose. As shown in Fig. 6, water sorption is very low for the coating formulation tested in the present study, and no significant alteration of sorption characteristics due to addition of nanocellulose was found. Thus, the water repellency of the coating formulation is not negatively affected by nanocellulose addition.

As reported elsewhere, the optical properties of a coating film on woody substrate are affected by nanocellulose addition in terms of reduced gloss due to increased surface roughness (Veigel et al. 2014). In the case of free-standing films, optical transparency is a good measure of potential effects of nanocellulose addition on coating optics. Overall, the wavelength-dependent pattern of light transmission through coating films is not gravely affected by nanocellulose addition as shown for selected films in Fig. 7. The impact on transparency is larger after addition of CNF than after the addition of $\mathrm{CNC}$ which can be explained by the overall larger particle size of CNF compared to CNC.

\section{Conclusion}

The results presented above demonstrate a clear potential for improving the static and dynamic mechanical properties of wood coating films by adding nanocellulosic fillers. Dispersion of nanocellulose in the waterborne formulation studied was 


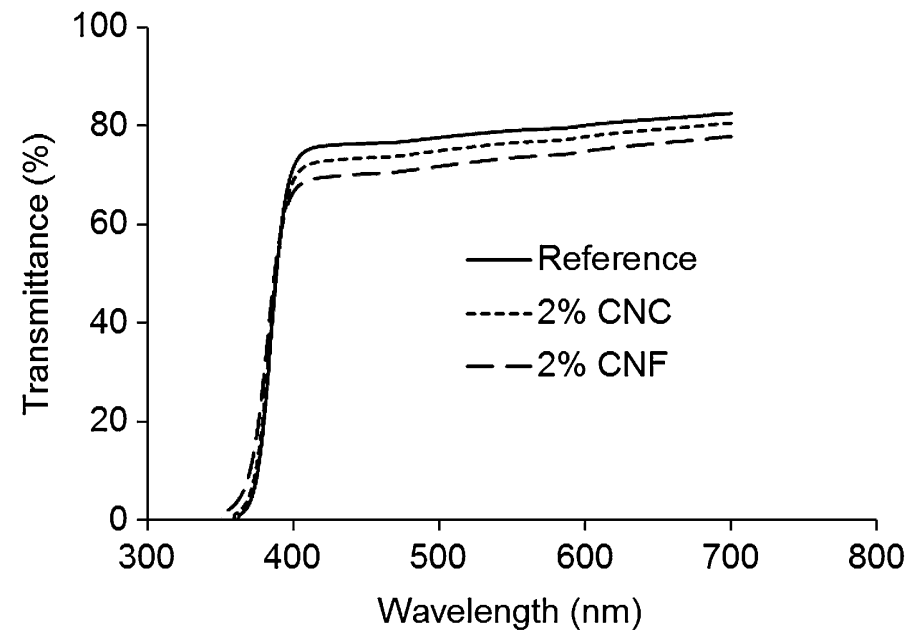

Fig. 7 Representative transmission spectra of free-standing coating films

successful without chemical modification, whereas chemical hydrophobization of nanocellulose deteriorated reinforcement efficiency. While the extensibility of coating films is impaired by network formation in microfibrillated cellulose, cellulose nanocrystals provide mechanical reinforcement while preserving extensibility. Water vapor sorption of coating films was not affected by nanocellulosic filler, whereas optical transparency was reduced significantly. In summary, unmodified nanocellulose with smaller aspect ratio than microfibrillated cellulose may be a promising option for reinforcing waterborne wood coatings.

Acknowledgements Open access funding provided by University of Natural Resources and Life Sciences Vienna (BOKU). The authors wish to thank Simon Lackner, Adler Werk Lackfabrik, for providing coating samples and for helpful discussion. Prof. Rupert Wimmer, BOKU Vienna, is gratefully acknowledged for providing access to DVS equipment.

Open Access This article is distributed under the terms of the Creative Commons Attribution 4.0 International License (http://creativecommons.org/licenses/by/4.0/), which permits unrestricted use, distribution, and reproduction in any medium, provided you give appropriate credit to the original author(s) and the source, provide a link to the Creative Commons license, and indicate if changes were made.

\section{References}

Ashori A, Babaee M, Jonoobi M, Hamzeh Y (2014) Solvent-free acetylation of cellulose nanofibers for improving compatibility and dispersion. Carbohydr Polym 102:369-375

Azizi Samir MAS, Alloin F, Dufresne A (2005) Review of recent research into cellulosic whiskers, their properties and their application in nanocomposite field. Biomacromolecules 6:612-626

Belbekhouche S, Bras J, Siqueira G, Chappey C, Lebrun L, Khelifi B, Marais S, Dufresne A (2011) Water sorption behavior and gas barrier properties of cellulose whiskers and microfibrils films. Carbohydr Polym 83:1740-1748 
Ben Elmabrouk A, Thielemans W, Dufresne A, Boufi S (2009) Preparation of poly(styrene-cohexylacrylate)/cellulose whiskers nanocomposites via miniemulsion polymerization. J Appl Polym Sci 114:2946-2955

Besbes I, Alila S, Boufi S (2011) Nanofibrillated cellulose from TEMPO-oxidized eucalyptus fibres: effect of the carboxyl content. Carbohydr Polym 84:975-983

Bulota M, Kreitsmann K, Hughes M, Paltakari J (2012) Acetylated microfibrillated cellulose as a toughening agent in poly(lactic acid). J Appl Polym Sci 126:E448-E457

Cetin NS, Tingaut P, Oezmen N, Henry N, Harper D, Dadmun M, Sebe G (2009) Acetylation of cellulose nanowhiskers with vinyl acetate under moderate conditions. Macromol Biosci 9:997-1003

Challener C (2015) Trends in interior wood coatings: tracking the shift from solvent to waterborne and UV. JCT Coatingstech 12:36-41

Eichhorn SJ, Dufresne A, Aranguren M, Marcovich NE, Capadona JR, Rowan SJ, Weder C, Thielemans W, Roman M, Renneckar S, Gindl W, Veigel S, Keckes J, Yano H, Abe K, Nogi M, Nakagaito AN, Mangalam A, Simonsen J, Benight AS, Bismarck A, Berglund LA, Peijs T (2010) Review: current international research into cellulose nanofibres and nanocomposites. J Mater Sci 45:1-33

Ernest-Saunders R, Pawlak JJ, Lee JM (2014) Properties of surface acetylated microfibrillated cellulose relative to intra- and inter-fibril bonding. Cellulose 21:1541-1552

Favier V, Canova GR, Cavaille JY, Chanzy H, Dufresne A, Gauthier C (1995) Nanocomposite materials from latex and cellulose whiskers. Polym Adv Technol 6:351-355

Grüneberger F, Künniger T, Zimmermann T, Arnold M (2014a) Nanofibrillated cellulose in wood coatings: mechanical properties of free composite films. J Mater Sci 49:6437-6448

Grüneberger F, Künniger T, Zimmermann T, Arnold M (2014b) Rheology of nanofibrillated cellulose/ acrylate systems for coating applications. Cellulose 21:1313-1326

Grüneberger F, Künniger T, Huch A, Zimmermann T, Arnold M (2015) Nanofibrillated cellulose in wood coatings: dispersion and stabilization of $\mathrm{ZnO}$ as UV absorber. Prog Org Coat 87:112-121

Habibi Y (2014) Key advances in the chemical modification of nanocelluloses. Chem Soc Rev 43:1519-1542

Higgins HG, Stewart CM, Harrington KJ (1961) Infrared spectra of cellulose and related polysaccharides. J Polym Sci 51:59-84

Hill CAS, Norton A, Newman G (2009) The water vapor sorption behavior of natural fibers. J Appl Polym Sci 112:1524-1537

Hincapie I, Künniger T, Hischier R, Cervellati D, Nowack B, Som C (2015) Nanoparticles in facade coatings: a survey of industrial experts on functional and environmental benefits and challenges. J Nanopart Res 17:287

Hischier R, Nowack B, Gottschalk F, Hincapie I, Steinfeldt M, Som C (2015) Life cycle assessment of facade coating systems containing manufactured nanomaterials. J Nanopart Res 17:68

Iotti M, Gregersen OW, Moe S, Lenes M (2011) Rheological studies of microfibrillar cellulose water dispersions. J Polym Environ 19:137-145

Jämsä S, Kataja K, Takala S, Putkisto K, Vastamäki P, Dyhr H (2011) Method for manufacturing paint or varnish. WO 2011124759 A1

Jonoobi M, Harun J, Mathew AP, Hussein MZB, Oksman K (2010) Preparation of cellulose nanofibers with hydrophobic surface characteristics. Cellulose 17:299-307

Kaboorani A, Auclair N, Riedl B, Landry V (2016) Physical and morphological properties of UV-cured cellulose nanocrystal (CNC) based nanocomposite coatings for wood furniture. Prog Org Coat 93:17-22

Klemm D, Kramer F, Moritz S, Lindstrom T, Ankerfors M, Gray D, Dorris A (2011) Nanocelluloses: a new family of nature-based materials. Angew Chem Int Ed 50:5438-5466

Landry V, Blanchet P (2011) Coatings containing nanocrystalline cellulose, processes for preparation and use thereof. WO 2011075837 A1

Lee K-Y, Aitomaki Y, Berglund LA, Oksman K, Bismarck A (2014) On the use of nanocellulose as reinforcement in polymer matrix composites. Compos Sci Technol 105:15-27

Li M-C, Wu Q, Song K, Lee S, Qing Y, Wu Y (2015) Cellulose nanoparticles: structure-morphologyrheology relationships. ACS Sustain Chem Eng 3:821-832

Littunen K, Hippi U, Saarinen T, Seppala J (2013) Network formation of nanofibrillated cellulose in solution blended poly(methyl methacrylate) composites. Carbohydr Polym 91:183-190

Liu Y, Soer W-J, Scheerder J, Satgurunathan G, Keddie JL (2015) Water vapor sorption and diffusion in secondary dispersion barrier coatings: a critical comparison with emulsion polymers. ACS Appl Mater Interfaces 7:12147-12157 
Moon RJ, Martini A, Nairn J, Simonsen J, Youngblood J (2011) Cellulose nanomaterials review: structure, properties and nanocomposites. Chem Soc Rev 40:3941-3994

Nikolic M, Lawther JM, Sanadi AR (2015) Use of nanofillers in wood coatings: a scientific review. J Coat Technol Res 12:445-461

Philipp C (2010) The future of wood coatings. Eur Coat J 1:1-6

Phipps J, Bourgoin S, Legrix AHR, Vinnicombe G (2013) Compositions for paint. WO 2013156223 A1

Pilotek S, Tabellion F (2005) Nanoparticles in coatings. Eur Coat J 4:170-176

Poaty B, Vardanyan V, Wilczak L, Chauve G, Riedl B (2014) Modification of cellulose nanocrystals as reinforcement derivatives for wood coatings. Prog Org Coat 77:813-820

Samios E, Dart RK, Dawkins JV (1997) Preparation, characterization and biodegradation studies on cellulose acetates with varying degrees of substitution. Polymer 38:3045-3054

Vardanyan V, Poaty B, Chauve G, Landry V, Galstian T, Riedl B (2014) Mechanical properties of UVwaterborne varnishes reinforced by cellulose nanocrystals. J Coat Technol Res 11:841-852

Vardanyan V, Galstian T, Riedl B (2015) Effect of addition of cellulose nanocrystals to wood coatings on color changes and surface roughness due to accelerated weathering. J Coat Technol Res 12:247-258

Veigel S, Grüll G, Pinkl S, Obersriebnig M, Müller U, Gindl-Altmutter W (2014) Improving the mechanical resistance of waterborne wood coatings by adding cellulose nanofibres. React Funct Polym 85:214-220

Vlad-Cristea MS, Landry V, Blanchet P, Ouellet-Plamondon C (2013) Nanocrystalline cellulose as effect pigment in clear coatings for wood. ISRN Nanomater. doi:10.1155/2013/930236

Weiss KD (1997) Paint and coatings: a mature industry in transition. Prog Polym Sci 22:203-245

Xie Y, Hill CAS, Jalaludin Z, Sun D (2011) The water vapour sorption behaviour of three celluloses: analysis using parallel exponential kinetics and interpretation using the Kelvin-Voigt viscoelastic model. Cellulose 18:517-530

Xu X, Liu F, Jiang L, Zhu JY, Haagenson D, Wiesenborn DP (2013) Cellulose nanocrystals vs. cellulose nanofibrils:aA comparative study on their microstructures and effects as polymer reinforcing agents. ACS Appl Mater Interfaces 5:2999-3009 